\title{
Design and Implementation of High Precision Digital Frequency Meter Based on C8051F020 Microcontroller
}

\author{
Li Su, Aiguo Shang, Jin Qin, Jingjing Yang \\ Xian Research Inst. of Hi-Tech Hong Qing Town, Xi'an, P.R. China \\ *18792994804@163.com
}

Keywords: C8051F020 microcontroller; digital frequency meter; high-speed comparator; direct measurement of the week; direct measurement frequency measurement

\begin{abstract}
For the oscilloscope, spectrum analyzer and other analog circuit equipment measured characteristics of the narrow frequency range, slow frequency measurement, the design of the digital frequency based on a C8051F020 microcontroller. Frequency meter hardware part mainly includes C8051F020 microcontroller, high speed comparator and TFT LCD screen. Comparator converts the enter signal into a square wave signal for the microcontroller count, TFT LCD screen display measurement frequency. Using a direct measurement frequency method combined with the direct measurement of the week in the control algorithm, which improved the accuracy of the measurement frequency. Experiments show that: the digital frequency meter can achieve the purpose of frequency measurement of digital, measurement frequency range of $1 \mathrm{~Hz} 4 \mathrm{MHz}$, and measurement frequency accuracy is high.

Frequency, is to describe the frequency of periodic movement of the physical quantity, easy to transmit, anti-interference ability, many engineering measurements are related to frequency, such as sound frequency, mechanical vibration, measurement speed ${ }^{[1]}$. With the advance of the digital process, the traditional frequency-based circuit and timing circuit design of the frequency meter in the speed, accuracy and other aspects of the trend gradually, but was based on ultra-large-scale integrated circuit technology developed from the digital frequency meter to replace it on a large scale. Digital frequency meter can not only solve the problem of low efficiency of analog circuit information processing and
\end{abstract}

transmission, but also improve the accuracy of frequency measurement ${ }^{[2]}$. Digital frequency meter is the use of digital circuits can be achieved on the cyclical changes in the signal frequency measurement of the instrument can be used to measure sine wave, rectangular wave, triangular wave and sharp pulse and other periodic signal frequency ${ }^{[3]}$.

At present, there are many ways to measure the frequency of the frequency meter. Commonly used methods include interpolation method, cursor method, precision frequency measurement method, frequency difference multiplication method, phase comparison method, direct frequency measurement method and direct measurement method. Interpolation method and cursor method for analog circuits, the circuit design is complex, poor stability ${ }^{[4-5]}$. Frequency difference method is mainly used for frequency stability measurement, anti-jamming performance is poor ${ }^{[6]}$. Equal precision frequency measurement method is very high accuracy, fast operation, but most of its use of the chip is a programmable logic device, programming more complex $^{[7]}$. Phase comparison method is mainly used for frequency accuracy and stability measurement,

measurement accuracy by the hardware equipment constraints $^{[8]}$. Based on the single-chip digital frequency meter commonly used frequency measurement method has direct frequency measurement method and direct measurement method, the direct measurement of the frequency range is limited, can only measure low frequency ${ }^{[9]}$. Direct frequency measurement is also limited by the frequency range, can only measure high frequency ${ }^{[10]}$.

This paper designs a digital frequency meter based on C8051F020 single chip microcomputer. The frequency measurement method of digital frequency meter adopts the direct frequency measurement method and the direct 
measurement method. The hardware module is based on C8051F020 single chip microcomputer as the core, thus overcomes the analog circuit frequency measurement range Limited, low rate of measurement problems.

\section{Fundamental}

The timing of the direct frequency measurement is shown in Figure 1. When the rising edge of the signal arrives, the gate time is on and the counter starts counting and stops counting when the gate is closed. If a signal in the $T$ seconds to repeat the change $N$ times, we can see that the frequency of the signal

$$
f_{x}=\frac{N}{T}
$$

Where: $f \leq f_{m}$ is the frequency to be measured, Hz; $T$ is the gate time, s; $N$ is the number of times the signal to be measured changes within the gate time $T$, dimensionless.

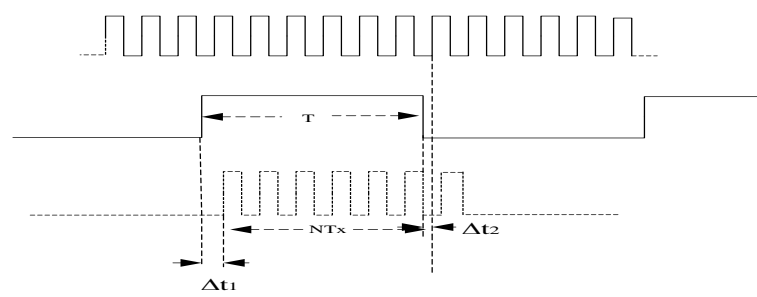

Figure 1. Direct frequency measurement

Direct measurement cycle timing shown in Figure 2. In the cycle of the signal to be measured within a cycle time, through the standard clock count to get the number of, then

$$
T_{x}=N_{s} T_{s}=\frac{N_{s}}{f_{s}}
$$

Where: $T_{x}$ is the signal period to be measured, s; $N_{s}$ is the number of times the standard clock signal changes in time, dimensionless; $T_{s}$ is the standard clock signal period, s; $f_{s}$ is the standard clock signal frequency, Hz.

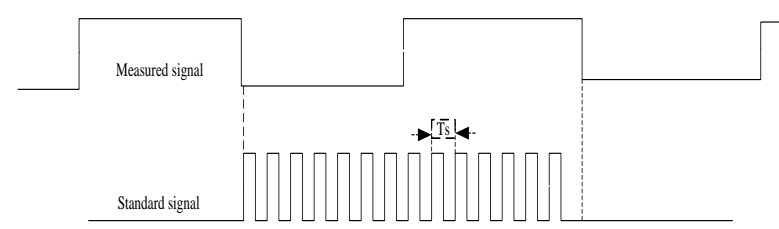

Figure 2. Direct measurement of weekly timing diagram When you choose to use the frequency measurement method in conjunction with the measurement method, set a frequency threshold $f_{m}$, when the measured signal frequency $f \geq f_{m}$, the choice of direct frequency measurement method. When the measured signal frequency $f \leq f_{m}$, the choice of direct measurement method. After several experimental tests, the frequency threshold is about $300 \mathrm{~Hz}$, the error is relatively small, so we take $f_{m}$ that is equal to $300 \mathrm{~Hz}$. First of all, the use of direct frequency measurement method for the initial frequency of the input signal, and then determine whether the measured signal frequency is less than $f_{m}$, if less than, then use the direct measurement of frequency measurement frequency.

\section{2 hardware design}

Digital frequency meter hardware from the power module, high-speed comparator module, C8051F020 microcontroller module, TFT LCD screen composition. The signal source is generated by a function signal generator, mainly generating square wave, sine wave for digital frequency meter measurement. The system block diagram is shown in Figure 3

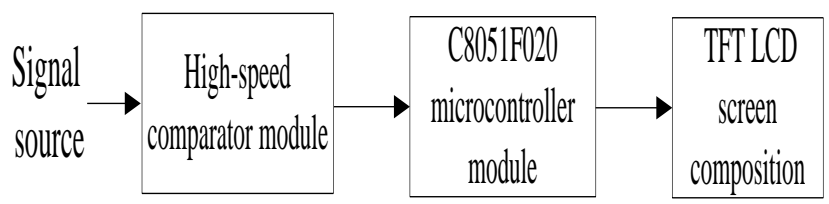

Figure 3. Digital frequency meter system block diagram

Power module. The entire system uses $+5 \mathrm{~V}$ switching power supply. C8051F020 microcontroller system and 
high-speed comparator requires $3.3 \mathrm{~V}$ power supply, which by VCC through the AS1117-3.3 low dropout voltage regulator chip, the circuit shown in Figure 4. In order to prevent crosstalk between different ground, to improve the anti-jamming capability, digital ground and analog use of zero ohm resistance to isolate. Since these two systems involve analog and digital power supplies, magnetic bead isolation between them is used to produce different numbers of analog and analog $3.3 \mathrm{~V}$ power supplies.

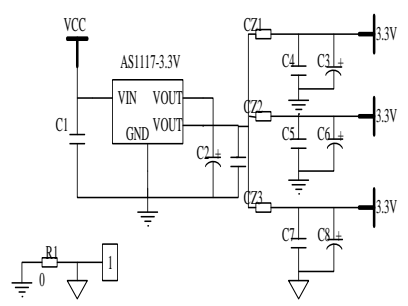

Figure 4. AS1117-3.3 V power supply

\section{High Speed Comparator Module.}

High-speed comparator module circuit using high-speed comparator chip TL3016, for the sine wave rectangle into a rectangular signal, the circuit shown in Figure 5. TL3016 low-frequency sine wave signal shaping, the output edge of a more serious jitter, affecting the measurement. So in the circuit to add positive feedback, accelerate the signal edge, while the formation of hysteresis, which can effectively eliminate jitter.

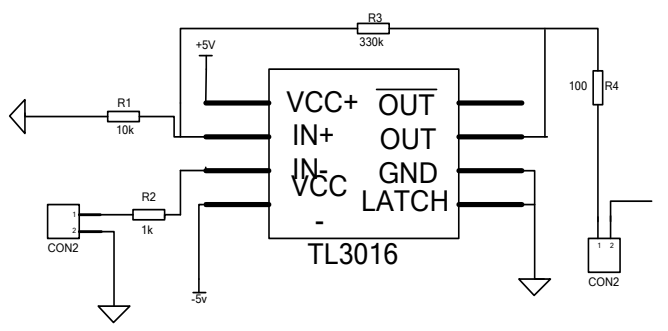

Figure 5. High Speed Comparator Module Circuit

C8051F020 single chip system. Digital frequency meter frequency measurement method is mainly achieved by the C8051F020 microcontroller chip timer and counter 2 and timer and counter 4 to complete. When the digital frequency meter uses the direct measurement method to measure the measured signal frequency, the timer and counter 2 count, timer and counter 4 timing, by setting the timer and counter 2 in the control register configuration bits, select the timer and counter 2 operates in 16-bit auto-reloaded counter mode, the timer and counter 4 operate in 16-bit auto-reload timer mode. When the digital frequency meter uses the direct frequency measurement method to measure the measured signal frequency, the timer and counter 2 timing, the timer and counter 4 count. By setting the timers in the control register of the timer and counter 2 , select the timer and counter 2 to operate in the 16-bit auto-reload timer mode, and the timer and counter 4 operate in the 16-bit auto-reload counter mode. When the timer and counter registers are overflowed, the timer and counter of the auto-reload mode set the timer overflow flag TF2 to "1". If the interrupt is enabled, an interrupt will be generated. At the time of overflow, the 16-bit count initial value in the two capture registers is automatically loaded into the timer and counter registers, and the timer and counter are restarted ${ }^{[11]}$. C8051F020 block diagram shown in Figure 6.

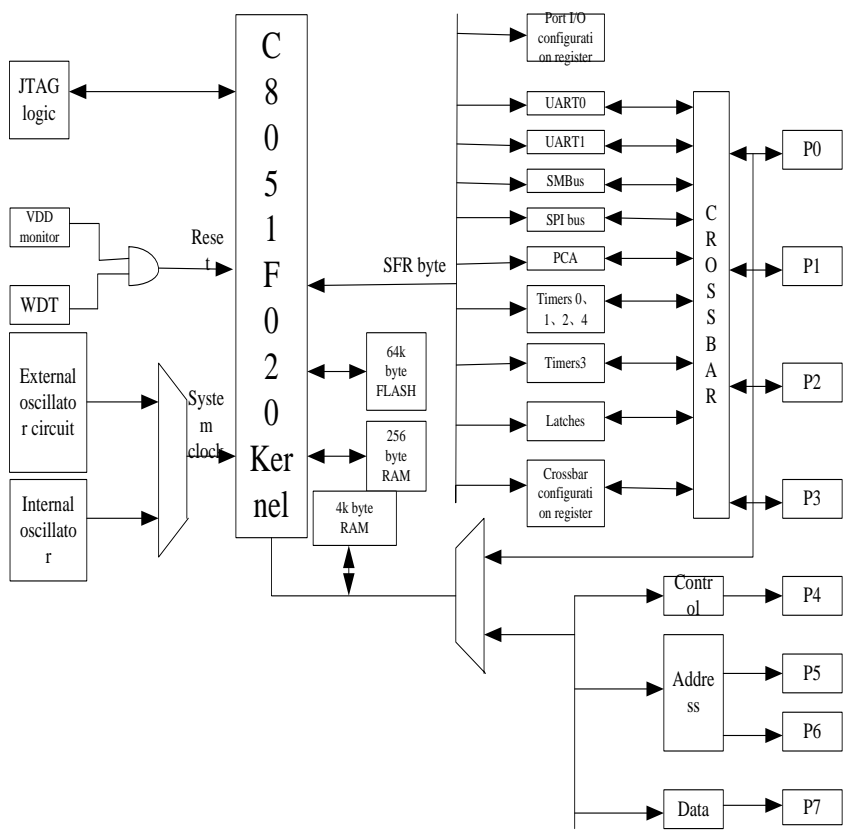

Figure 6. C8051F020 block diagram

TFT LCD screen. Digital frequency meter TFT LCD screen is mainly used to display the frequency and picture, and drive control TFT LCD screen is the integrated circuit chip SPFD5408. When the TFT LCD shows the frequency, set the display area by setting the window horizontal start position setting register of the integrated circuit chip SPFD5408, the window horizontal end position setting register, the window vertical start 
position setting register, the window vertical end position setting register The address of the display data memory can be set to horizontal or vertical. When the TFT LCD display picture, TFT LCD screen with window display and coordinates from the show. The window display needs to set the size of the image display area and the scanning direction. TFT LCD screen resolution is $320 \times$ 240 , by setting the $\mathrm{X}$ coordinates, $\mathrm{Y}$ coordinates, open up a window, and then write

data to them, the window within the address will be set in accordance with the direction of self-increment.

\section{System software design}

The system is initialized first, and when the signal is input, the frequency is first measured by the frequency measurement method, and then the measurement is made by the direct frequency measurement method for signals greater than $300 \mathrm{~Hz}$. For less than or equal to $300 \mathrm{~Hz}$ signal, measured by the measurement method, the main program flow chart shown in Figure 7.

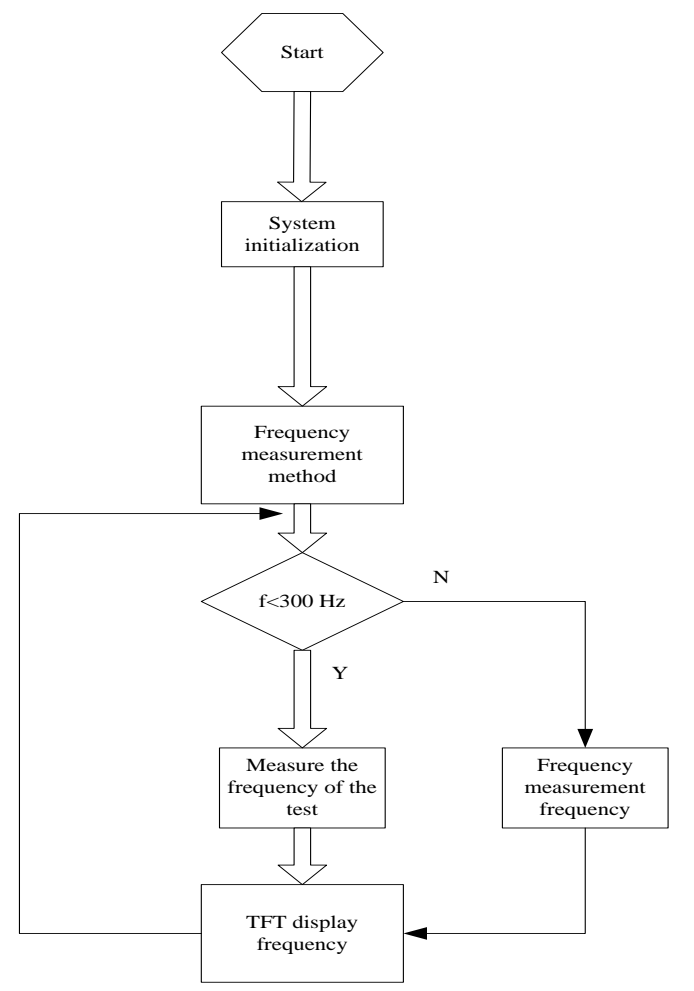

Figure 7. Main program flow chart

\section{Experimental test}

4.1 Experimental results. With the signal generator to generate the input signal, after the high-speed comparator TL3016, you can test the sine wave, square wave, triangular wave and other periodic waveform frequency values. When the input waveform is sine wave signal and square wave signal, their measured values and errors are shown in Table 1, Table 2, respectively.

Table I. sine wave measurement data table
$2.5 \mathrm{~V}$ sine wave
$2.8 \mathrm{~V}$ sine wave

\begin{tabular}{cccccc}
\hline Actual & Measu & Relativ & $\begin{array}{c}\text { Actual } \\
\text { value }\end{array}$ & $\begin{array}{c}\text { Mea } \\
\text { sureme }\end{array}$ & $\begin{array}{c}\text { Relativ } \\
\text { e error }\end{array}$ \\
$\mathrm{Hz}$ & $\mathrm{s} / \mathrm{Hz}$ & e error & $\mathrm{Hz}$ & $\mathrm{nts} / \mathrm{Hz}$ & \\
\hline 1 & 1.1 & 0.1 & 1 & 1.2 & 0.2 \\
101 & 114 & 0.13 & 101 & 112 & 0.109 \\
1008 & 1016 & 0.008 & 1008 & 1013 & 0.005 \\
1523 & 1542 & 0.012 & 1523 & 1538 & 0.0098 \\
5010 & 5022 & 0.012 & 5010 & 5015 & 0.001 \\
10000 & 10001 & 0.0001 & 10000 & 10003 & 0.0003 \\
15002 & 15012 & 0.0006 & 15002 & 15010 & 0.0005 \\
& & 7 & & & 3 \\
20012 & 20043 & 0.0015 & 20012 & 20033 & 0.001 \\
30008 & 30013 & 0.0001 & 30008 & 30015 & 0.0002 \\
10000 & 10000 & 0.0000 & 10000 & 10000 & 0.0000 \\
0 & 4 & 4 & 0 & 5 & 5 \\
10000 & 10001 & 0.0001 & 10000 & 10001 & 0.0001 \\
00 & 23 & 2 & 00 & 34 & 3 \\
40000 & 40001 & 0.0000 & 40000 & 40001 & 0.0000 \\
00 & 42 & 36 & 00 & 38 & 35 \\
\hline
\end{tabular}


Table II. square wave measurement data table

\begin{tabular}{|c|c|c|c|c|c|}
\hline \multicolumn{3}{|c|}{$2.5 \mathrm{~V} \mathrm{~s}$} & \multicolumn{3}{|c|}{$2.8 \mathrm{~V}$ square wave } \\
\hline $\begin{array}{c}\text { Actual } \\
\text { value } \\
/ \mathrm{Hz}\end{array}$ & $\begin{array}{c}\text { Measur } \\
\text { ements } \\
/ \mathrm{Hz}\end{array}$ & $\begin{array}{l}\text { Relativ } \\
\text { e error }\end{array}$ & $\begin{array}{c}\text { Actual } \\
\text { value } \\
/ \mathrm{Hz}\end{array}$ & $\begin{array}{r}\text { Mea } \\
\text { sureme } \\
\text { nts } / \mathrm{Hz}\end{array}$ & $\begin{array}{l}\text { Relativ } \\
\text { e error }\end{array}$ \\
\hline 1 & 1 . & & 1 & 1.1 & \\
\hline 101 & ( & & 101 & 106 & \\
\hline 1008 & 1003 & 6 & 1008 & 1001 & $\begin{array}{c}0.0069 \\
4\end{array}$ \\
\hline 1523 & 1526 & $\begin{array}{c}0.0019 \\
7\end{array}$ & 1523 & 1514 & $\begin{array}{c}0.0059 \\
1\end{array}$ \\
\hline 5010 & 5018 & $\begin{array}{c}0.0016 \\
0\end{array}$ & 5010 & 5002 & $\begin{array}{c}0.0016 \\
0\end{array}$ \\
\hline 10000 & 100 & 0.0009 & 10000 & 100 & 0.0008 \\
\hline 15002 & 150 & $\begin{array}{c}0.0003 \\
3\end{array}$ & 15002 & 15007 & $\begin{array}{c}0.0003 \\
3\end{array}$ \\
\hline 20012 & 200 & $\begin{array}{c}0.0007 \\
0\end{array}$ & 012 & 030 & $\begin{array}{c}0.0009 \\
0\end{array}$ \\
\hline 30008 & 3001 & $\begin{array}{c}0.0003 \\
7\end{array}$ & 008 & 30023 & $\begin{array}{c}0.0005 \\
0\end{array}$ \\
\hline 100000 & 100023 & $\begin{array}{c}0.0002 \\
3\end{array}$ & 100000 & 100011 & $\begin{array}{c}0.0001 \\
1\end{array}$ \\
\hline $\begin{array}{c}100000 \\
0\end{array}$ & $\begin{array}{c}100010 \\
4\end{array}$ & $\begin{array}{c}0.0001 \\
0\end{array}$ & $\begin{array}{c}100000 \\
0\end{array}$ & $\begin{array}{c}100009 \\
8\end{array}$ & $\begin{array}{c}0.0000 \\
98\end{array}$ \\
\hline 400000 & 40002 & & 40000 & 400019 & 0.0000 \\
\hline 0 & 3 & 68 & 0 & 9 & 50 \\
\hline
\end{tabular}

4.2 Result analysis. From the above measurement data available, through the direct frequency measurement method and the direct measurement method combined with the input signal for the sine wave or square wave, the frequency range of $1 \mathrm{~Hz} \sim 4 \mathrm{MHz}$. In the low-frequency measurement, the relative error of the measured value is too large, the analysis of the rising edge or falling edge of the pulse, the high-speed comparator TL3016 after shaping the square wave signal, there will be up and down jitter, and the program set Is falling edge capture. Thus, if an interrupt is triggered on the rising edge of the jitter, the interrupt is triggered on the next true falling edge, resulting in a larger measurement error. In order to trigger the capture interrupt in the service routine, to determine whether the true falling edge. Can be in the interrupt service routine first delay for some time, and then determine the C8051F020 input signal pin $\mathrm{P}^{\wedge} \wedge 0$ is not low. If so, it is judged as a true falling edge, which reduces the relative error at low frequency measurements.

\section{Conclusion}

This paper starts from the function of digital frequency meter, analyzes the commonly used frequency measurement method of digital frequency meter, uses the integrated circuit chip C8051F020 as the control module, the high speed comparator as the waveform conversion module, uses the TFT liquid crystal display to display, and the method of direct measurement of the method, the low frequency, intermediate frequency and high frequency are measured under different voltages, and the frequency range of $1 \mathrm{~Hz} \sim 4 \mathrm{MHz}$ is preliminarily designed, which is feasible and practicable.

\section{References}

[1] Fu J, Chen X, Huang Y. 2013. Compression Frequency Choice for Compression Mass Gauge Method and Effect on Measurement Accuracy[J]. Microgravity Science and Technology, 25(4): 213-223.

[2] Watanabe T, Terasawa T. 2013. All-digital A/D converter TAD with high-resolution and low-power for sensor/RF digitization[J]. Analog Integrated Circuits and Signal Processing, 77(3): 449-457.

[3]Haopeng Wang, Zejian Liu. 2011. Design and Implementation of Simple Digital Frequency Meter [J]. Journal of Sichuan Wa, 32(9): 85-107

[4]Jifei Pan, Qiuxi Jiang. 2007. BI Da-ping.Modeling interpolation and its measurement error analysis [J]. Electro-Optic and Control, 14(01): 148-150.

[5]Zhiwen Zhang. 2012. wang qing Design of High Accuracy Frequency Measurement System Based on Cursor Method [J]. Science Technology and Engineering, 31(12): 8229-8234.

[6]Li Yang. 2012. The development of high precision time domain frequency stability measuring instrument using frequency difference multiplication method [D]. Wuhan: Wuhan University of Technology, 47-48.

[7]Jieling Wang, Qi Li, Wang Dao Ping. 2016. Broadband FPGA-based digital frequency meter design and implementation [J]. Science and Innovation, 15: 09-10.

[8]Zhixun Xu. 2002. Principle and Error Analysis of Phase Comparison Method [J]. Measurement Technology, 06: 14-17.

[9]Shaohua Hu, Zhongchun Cai, Ming Jia. 2012. Design of Frequency and Duty Cycle Detection Circuit Based on Measurement Method [J]. Machinery and Electronics, 21: 90-134. 
[10]Lixin Chen. 2012. Design of Digital Frequency Meter Based on CPLD Direct Frequency Measurement [J]. Xuzhou Institute's Journal of Technology, 03: 22-28.

[11]Zhuojin Pan, Shi Guo Jun. 2002. The principle and application of C8051Fxxx high speed SOC single chip microcomputer $[\mathrm{M}]$. Beijing: Beijing University of Aeronautics and Astronautics Press. 